\title{
Los QALYs y DALYs como indicadores sintéticos de salud
}

\author{
NELSON ALVIS ${ }^{1, a}$, MARÍA TERESA VALENZUELA ${ }^{2, b}$

\section{QALYs and DALYs as synthetic indicators of health}

This article is a revision of the genesis and use of the health synthetic indicators QALYs and DALYs. The chronology of their appearance is shown and similarities and differences between them stated. DALYs (Disability-Adjusted Life-Years) correspond to years of life that are adjusted by a certain level of disability experienced during a particular period of time. DALYs, by studying disease burden, summarize the impact of mortality and disability related to specific disease in different communities. On the other hand, QALYs (Quality-Adjusted Life-Years) summarize health results in an indicator that combines the number of years lived with the quality of life experienced over those years. QALYs and DALYs play a crucial role as results measures in economic evaluation studies (cost-effectiveness and cost-utility analysis) measuring the impact of actions or specific interventions in the reversion of disease burden (DALYs) and/ or the improvement of the quality of life and life expectancy of patients (QALYs). Their generic character allows the comparison of the impact of health interventions for different diseases.

(Rev Med Chile 2010; 138 (Supl 2): 83-87).

Key words: DALYs, QALYs, disease burden, economic evaluation.

\section{RESUMEN}

En el presente artículo se revisa la génesis y uso de los indicadores sintéticos de salud QALYs y DALYs. Se muestra la cronología de su aparición y se establecen las semejanzas y diferencias entre los mismos. Los DALYs (Disability Adjusted Life Years) corresponden a los años de vida ajustados por la Discapacidad o AVADs por su sigla en español. Los DALYs resumen, mediante estudios de carga de la enfermedad, el impacto de la mortalidad y discapacidad asociada a enfermedades especificas, en distintas comunidades; en cambio los QALYs (Quality Adjusted Life Years) sintetizan los resultados de salud en un indicador que combina el número de años vividos con la calidad de vida experimentada durante esos años. QALYs y DALYs cumplen hoy un papel esencial como medidas de resultado en los estudios de evaluación económica (análisis de costo efectividad y costo utilidad) midiendo el impacto de acciones o intervenciones especificas para revertir la carga de la enfermedad (DALYs) y/o mejorar la calidad y esperanza de vida de pacientes (QALYs). Su carácter genérico permite comparar el impacto de intervenciones de salud entre diferentes enfermedades.

Palabras clave: DALYs, QALYs, Carga de enfermedad, evaluación económica en salud.

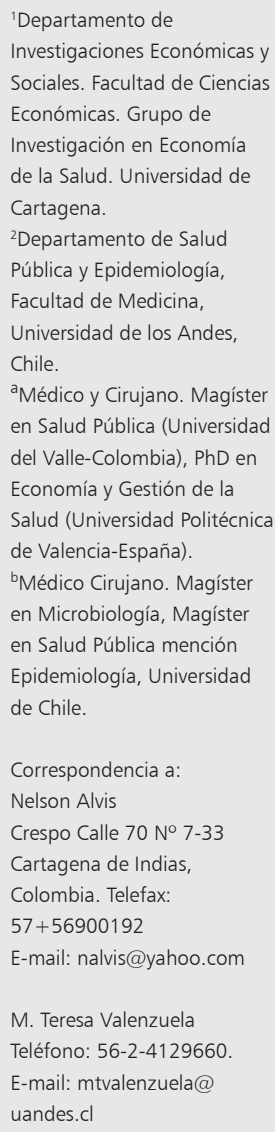


E $\mathrm{n}$ las primeras mediciones del impacto de la enfermedad en la salud de las poblaciones, sólo se estudiaba la mortalidad (por ejemplo, años de vida potencialmente perdidos, tasa de mortalidad infantil, etc). Así, sólo se analizaban las principales causas de muerte, ignorando una cantidad importante de discapacidad asociada a la enfermedad. Durante los años 1960 y 1970 se iniciaron los esfuerzos por medir los resultados de salud más allá de la mortalidad y hoy se cuenta con una enorme literatura sobre la medición de la Calidad de Vida Relacionada con la Salud $(\mathrm{CVRS})^{1}$. Actualmente, se dispone de tres grupos principales de instrumentos para comparar la salud de las personas entre los cuales se cuentan los que combinan la mortalidad y la morbilidad ${ }^{2}$. Los indicadores sintéticos de la salud, al combinar información sobre la mortalidad y la calidad de vida relativa a la salud, proporcionan un valioso instrumento para resumir, controlar y comparar la salud de las poblaciones, así como para determinar la importancia relativa de las diversas causas de las diferencias halladas ${ }^{3}$. Con relación a los QALYs y DALYs este artículo busca discutir las siguientes preguntas: ¿Qué son? ¿Para que se usan? ¿Cuándo utilizarlos?

\section{QALYs como unidades de medición en salud}

El término 'Quality Adjusted Life Year' (QALY) fue usado por primer vez en 1976 por Zeckhauser y Shepard ${ }^{4}$ como medida de resultado de salud que combina duración y calidad de vida. Sin embargo, su gestación como concepto se inicia a principio de los años 70 en el desarrollo de un índice de estado de salud (health status index) (5, $^{5}$ aunque ya en 1968 se había usado una medida de ajuste subjetivo para la calidad de la vida en un análisis de costo efectividad del tratamiento de la enfermedad renal crónica ${ }^{7}$.

El QALY es una unidad de medida de las preferencias de los individuos respecto a la calidad de vida que se ha producido mediante una intervención sanitaria, combinada con los años ganados respecto de un determinado estado de salud. Por esto los QALYs son altamente valorados como un ingrediente básico en la evaluación económica de intervenciones de salud, para la toma de decisiones.

Para su cálculo se combinan dos variables: calidad de vida y cantidad de vida. Para ello se necesitan ponderaciones de la Calidad de Vida Relacionada con la Salud (CVRS) de cada estado de salud considerado en el análisis ${ }^{8}$. Para obtener los pesos de CVRS se pueden utilizar diferentes métodos: Juego estándar, Escala visual analógica, Equivalencia temporal o Equivalencia de personas. Una alternativa muy atractiva y ampliamente usada ahora es evitarse la tarea de medir y usar sistemas creados para clasificar estado de salud multi-atributos ya ponderados. Los principales disponibles en la actualidad son: Calidad de Bienestar (QWB), el Índice de Utilidades de Salud (HUI) y el EuroQol (EQ-5D) ${ }^{8}$.

En cuanto al uso de los QALYs, esta medida de resultado pasó a ser la medida estándar para los estudios de costo utilidad. En 1992, Gerard publica una revisión en la cual incluye 51 estudios de evaluación económica en la cual se usan los QALYs como medida de resultado. Posteriormente, su uso ha sido aceptado ampliamente en los estudios de costo efectividad ${ }^{8-10}$. Los QALYs se han convertido en fórmula que soluciona la falta de comparabilidad de resultados al momento de evaluar la utilidad de diferentes programas de salud aplicados en distintas áreas sanitarias.

\section{Los DALYs como unidades de medición en salud}

El diseño de indicadores sintéticos de salud se inicia con Sanders ${ }^{11}$ en los años 60 y continúa con Sullivan ${ }^{12}$, quien en los 70 definió los métodos para el cálculo de las expectativas de vida -EV-con y sin discapacidad. Como lo establece Schopper ${ }^{13}$, en 1993 se consolidan los intentos previos por construir un indicador que resumiese las medidas de mortalidad y morbilidad, al usar los DALYs como un indicador sintético de salud en el marco del "Informe de Desarrollo Mundial 1993: Invertir en Salud"14. Los DALYs combinan los años potenciales de vida perdidos (APVP) más los años vividos con discapacidad (AVD) dentro del proceso salud enfermedad. Su principal utilidad se refiere a los estudios de carga de la enfermedad, los cuales se definen como un indicador que permite medir las pérdidas de salud que para una población representan la mortalidad prematura y la discapacidad asociada a las enfermedades ${ }^{15}$. En dichos estudios, los problemas de salud son clasificados 
en tres grupos exhaustivos y excluyentes: Grupo I Enfermedades infecciosas, maternas, perinatales y nutricionales; Grupo II Enfermedades no transmisibles y Grupo III Accidentes y lesiones. A su vez, estos grupos se dividen en una veintena de categorías también exhaustivas y excluyentes. Por último, hay un tercer y cuarto nivel (subcategorías y subgrupos) no necesariamente exhaustivos y pueden variar en función de la distintas patologías de la población estudiada.

\section{¿Cómo se calculan los DALYs?}

Los Años de Vida Ajustados por Discapacidad (AVAD) son el resultado de agregar los Años Potenciales de Vida Perdidos (APVP) más los Años Vividos con Discapacidad (AVD). Generalmente los años de vida perdidos por cada muerte se establecen en función de la Esperanza de Vida a la edad de cada muerte obtenida de tablas de vida estándar de baja mortalidad ${ }^{16}$. Un DALY representa la pérdida de un año que equivale a un año de completa salud, o equivalente a la pérdida de un año $100 \%$ saludable.

A nivel agregado los AVD dependen de los siguientes parámetros:

$$
\mathrm{AVD}=\sum_{0}^{\mathrm{L}} \mathrm{N}_{\mathrm{j}} * \mathrm{I} * \mathrm{~T}_{\mathrm{j}} * \mathrm{D}
$$

donde:

AVD: total Años Vividos con Discapacidad.

$\mathrm{N}_{\text {: }}$ : Población susceptible de enfermar a cada $\operatorname{edad}^{\prime}(0$ - L).

I: Incidencia de la enfermedad a cada edad $(0-\mathrm{L})$.

$\mathrm{T}_{\text {: }}$ D Duración media de la enfermedad desde la edad de inicio $(0-\mathrm{L})$.

D: Valor que pondera la discapacidad entre valores de " $0=$ salud perfecta" y " $1=$ máxima discapacidad o equivalente al estado de muerte". El procedimiento adoptado para establecer las escalas de severidad que sirven para ponderar los distintos estados de salud o enfermedades es el de Equivalencia de personas ${ }^{17}$.

De acuerdo a la metodología original, el cálculo de los DALYs requiere ponderar los años vividos a cada edad (Figura 1), lo cual se justifica por el hecho de que en los años centrales de la vida, las personas no sólo sostienen económicamente a los más jóvenes y ancianos, sino que realizan una serie de funciones sociales que repercuten favorablemente en la salud de los individuos de otras edades. Dicha función social se valora mediante la siguiente fórmula: $\mathrm{y}=\mathrm{c}^{\star} \mathrm{e}^{\mathrm{x}}$, donde $\mathrm{y}=$ valor de un año de vida a cada edad, $\mathrm{c}=0,16458$, e $=$ la base de los logaritmos neperianos, $\mathrm{x}=$ edad, $\mathrm{y}=0,04$ son constantes. Para un mejor entendimiento de la metodología y calculo de los DALYs se recomienda revisar a Fox-Rushby y Hanson ${ }^{18}$.

En el proceso de estimación de los DALYs, como medida de la carga de enfermedad, existen aspectos controversiales respecto a la selección de la tabla de vida, la diferencia entre las EV de hombres y mujeres, la cercanía temporal a la medición y a la ponderación de la severidad de los estados de salud discapacitantes.

Para comprender mejor estas controversias, si se seleccionan tablas con alta EV en relación al país donde se aplique, se genera un sesgo de ponderación de años por muerte prematura en individuos que mueren de acuerdo a otra realidad. Inicialmente los DALYs fueron propuestos con EV estándar (80 años), pero desde hace bastante tiempo se ha hecho una norma usar EV y tablas de vida específicas a cada país. Dado que las EV por sexo son mayores en las mujeres, las muertes a igual edad por sexo, contribuyen con diferentes APVP. En cuanto a la temporalidad, las perdidas de salud son más importantes cuanto más cercanas están al momento presente. Así, los AVD y APVP más próximos en el tiempo tendrán mayor valor. Sin embargo, estos valores decrecen en función de una tasa de descuento; para los estudios de carga

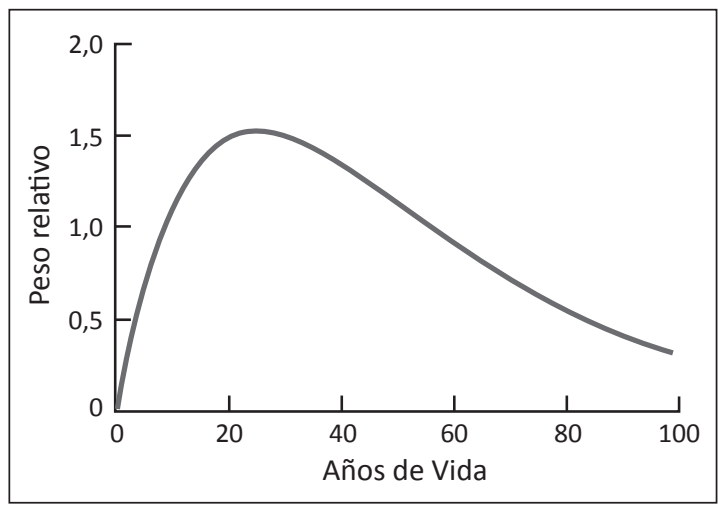

Figura 1. Valor relativo de un año de la vida vivido a diversas edades, según lo ponderado en DALYs. 
Los QALYs y DALYs como indicadores sintéticos de salud - N. Alvis et al

Tabla 1. Defunciones y carga de morbilidad según la causa: Países de ingreso bajo y mediano, países de ingreso alto y el mundo entero, 2001

\begin{tabular}{|lccc|}
\hline Grupos de causas & $\begin{array}{c}\text { Países de ingreso } \\
\text { bajo y mediano } \\
\text { AVAD en miles (\%) }\end{array}$ & $\begin{array}{c}\text { Países de ingreso } \\
\text { alto } \\
\text { AVAD en miles (\%) }\end{array}$ & $\begin{array}{c}\text { Mundo entero } \\
\text { AVAD en miles (\%) }\end{array}$ \\
$\begin{array}{l}\text { I. Enfermedades transmisibles, } \\
\text { trastornos maternos y del período } \\
\text { perinatal y carencias nutricionales }\end{array}$ & $552.376(39,8)$ & $8.561(5,7)$ & $560.937(36,5)$ \\
\hline $\begin{array}{l}\text { II. Enfermedades no transmisibles } \\
\text { III. Traumatismos }\end{array}$ & $678.483(48,9)$ & $129.356(86,7)$ & $807.839(52,6)$ \\
\hline
\end{tabular}

Fuente: Tomado de López AD, y col.

de enfermedad se establecen de manera arbitraria en el $3 \%$.

Los DALYs cuyo uso básico ha sido como medida de los estudios de carga de enfermedad, cada vez son más utilizados como medida de resultado en los análisis de costo efectividad. En tal sentido, usando como base los índices de discapacidad del estudio de carga de la enfermedad de Murray $1996^{19}$, es posible recalcular los DALYs, adaptando las variables (por ejemplo: tasa de descuento, Esperanza de Vida y ajuste por años) a las necesidades y criterios metodológicos locales.

Un ejemplo de aplicación en los DALYs es la medición de la carga mundial de morbilidad y de los factores de riesgo, realizada por López $\mathrm{A}, \mathrm{y} \mathrm{col}^{20}$, en la que se muestra una diferencia clara entre los países, clasificados según su nivel de ingreso, en la distribución de la carga de morbilidad por grupos de causas. Como era de esperarse, las causas del grupo I son más prevalentes entre los países de bajos y medianos ingresos (Tabla 1).

En síntesis, los DALYs se han consolidado como indicadores sintéticos de salud que resumen y permiten comparar el impacto de los riesgos (mortales y no mortales) entre comunidades mediante estudios de carga de enfermedad. Los QALYs cumplen hoy un papel esencial como medida de resultado en los estudios de evaluación económica (análisis de costo efectividad y costo utilidad).

Como medida de evaluación económica los evaluadores determinan los DALYs prevenidos, mediante la aplicación de intervenciones específicas que reducen la discapacidad y/o mortalidad, mientras que las QALYs estiman el impacto de una intervención en incrementar la calidad y/o
EV (QALYs ganadas). Tomando en consideración las diferencias de QALYS y DALYS en evaluación económica se puede decir que estos métodos constituyen la visión opuesta del mismo hecho ${ }^{21}$. Mientras DALYs son un mal que debe ser minimizado QALYs son un bien que debe ser maximizado.

Aspectos prácticos en investigación han llevado a incrementar el uso de los DALYs en países en desarrollo donde resulta caro establecer estudios clínicos para medir los parámetros necesarios para estimar QALYs. Por otro lado, países desarrollados como el Reino Unido, EEUU y Canadá entre otros tienden a intensificar (y recomendar) el uso de QALYs debido a su supuesta superioridad metodológica.

\section{Referencias}

1. Gold MR, Stevenson D, Fryback DG. HALYS and QALYS and DALYS, Oh My: similarities and differences in summary measures of population Health. Annu Rev Public Health 2002; 23: 115-34.

2. Fox-Rushby JA. The relationship between health economics and health-related quality of life. En: Orley, J., Kuyken, W., eds. Quality of life assessment International perspectives. Berlin and Heidelberg: Springer-Verlag. 1994.

3. Murray CJ, Salomon JA, Mathers C. A critical examination of summary measures of population health. Bull World Health Organ 2000; 78: 981-94.

4. Zeckhauser R, Shepard DS. Where now for saving lives? Law and Contemporary Problems. 1976; 40: 5-45.

5. Fanshel S, Bush JW. A health-status index and its application to health-services outcomes. Operations Research 1970; 18: 1021-66. 
Los QALYs y DALYs como indicadores sintéticos de salud - N. Alvis et al

6. Torrance GW. A generalized cost-effectiveness model for the evaluation of health programs. . Hamilton, Ontario: McMaster University, Faculty of Business.; 1970 Contract No.: Document Number|.

7. Klarman HE, Francis JOS, Rosenthal GD. Cost-effectiveness analysis applied to the treatment of chronic renal disease. Medical Care 1968; 6: 48-54.

8. Drummond M, O’Brien B, Stoddart G, Torrance G. Methods for the economic evaluation of health care programs. Third Edition ed. Oxford GB: Oxford Medical Publications; 2005.

9. Siegel JE, Weinstein MC, Russell LB, Gold MR. Recommendations for reporting cost-effectiveness analyses. Panel on Cost-Effectiveness in Health and Medicine. JAMA 1996; 276: 1339-41.

10. Weinstein MC, Siegel JE, Gold MR, Kamlet MS, Russell LB. Recommendations of the Panel on Cost-effectiveness in Health and Medicine. JAMA 1996; 276: 1253-8.

11. Sanders BS. Measuring Community Health Levels. Am J Public Health Nations Health 1964; 54: 1063-70.

12. Sullivan DF. A single index of mortality and morbidity. HSMHA Health Rep 1971; 86: 347-54.

13. Schopper D, Pereira J, Torres A, Cuende N, Alonso M, Baylin A, et al. Estimating the burden of disease in one Swiss canton: what do disability adjusted life years
(DALY) tell us? Int J Epidemiol 2000; 29: 871-7.

14. Musgrove P. Investing in health: the 1993 World Development Report of the World Bank. Bull Pan Am Health Organ 1993; 27: 284-6.

15. Murray CJ, López AD. Evidence-based health policy-lessons from the Global Burden of Disease Study. Science 1996; 274: 740-3.

16. Coale A, Guo G. Revised regional model life tables at very low levels of mortality. Popul Index. 1989; 55: 61343.

17. Murray CJ, López AD. Quantifying disability: data, methods and results. Bull World Health Organ 1994; 72: 481-94.

18. Fox-Rushby JA, Hanson K. Calculating and presenting disability adjusted life years (DALYs) in cost-effectiveness analysis. Health Policy Plan 2001; 16: 326-31.

19. Murray C, López A. The global burden of disease. Cambridge, MA: Harvard University Press; 1996.

20. López AD, Mathers CD, Ezzati M, Jamison DT, Murray CJ. Global and regional burden of disease and risk factors, 2001: systematic analysis of population health data. Lancet 2006; 367: 1747-57.

21. Arnesen T, Nord E. The value of DALY life: problems with ethics and validity of disability adjusted life years. BMJ 1999; 319: 1423-5. 